\title{
La conformation des protéines lors de leur translocation dans les membranes biologiques
}

Le ciblage des protéines et leur translocation dans les membranes des différents compartiments intracellulaires est un phénomène commun à tous les êtres vivants, des bactéries aux humains ( $\mathrm{m} / \mathrm{s}$ suppl. au $n^{\circ} 10$, vol. 4, p.6). Ces mécanismes sont nécessaires pour assurer le maintien de la compartimentalisation cellulaire et le bon fonctionnement des étapes initiales de la sécrétion des protéines. Le problème majeur posé par ce type de mécanisme est que les protéines ne peuvent traverser les membranes biologiques tout en gardant leur structure tridimensionnelle native. Dans le cas des protéines qui sont insérées de façon co-traductionnelle dans la membrane du réticulum endoplasmique, la translocation se passe à un moment où la protéine, en voie d'élongation n'a pas encore acquis sa conformation finale. $\mathrm{La}$ présence transitoire d'un peptide signal additionnel, situé généralement à l'extrémité $\mathrm{NH}_{2}$-terminale de ces protéines, pourrait également contribuer à l'établissement d'une conformation apte à favoriser la translocation $\left(\mathrm{m} / \mathrm{s} n^{\circ} 6\right.$, vol. 2 , p. 341). Il semble donc que pour cette classe de protéines, ce problème conformationnel ait trouvé une solution relativement simple. Comment, cependant, peut-on expliquer le phénomène dans le cas des protéines, telles que les protéines mitochondriales, pour lesquelles la translocation est clairement post-traductionnelle?

Au cours des quelques derniers mois, notre connaissance du mécanisme d'importation des protéines mitochondriales a grandement progressé. Le problème de la conformation semble avoir trouvé un début de réponse, et les résultats récemment obtenus à ce sujet permettront probablement de proposer bientôt un phénomènes de ciblage vers d'autres organites intracellulaires.

On sait que la plupart des protéines mitochondriales sont codées par le génome nucléaire et synthétisées, généralement sous la forme de précurseurs, sur les ribosomes libres. Il est également bien connu que leur translocation au travers des membranes mitochondriales se fait de façon post-traductionnelle et dépend le plus souvent de la présence d'une séquence additionnelle située à leur extrémité $\mathbf{N}$-terminale. Cette séquence est éliminée quand la protéine a rejoint sa destination finale ( $m / s n^{\circ} 4$, vol. 4, p. 258).

Afin d'identifier les changements de conformation qui accompagnent l'importation des protéines dans les mitochondries, l'équipe de G. Schatz a utilisé la dihydrofolate réductase de souris (DHFR). La DHFR est une protéine non mitochondriale que l'on peut toutefois cibler vers la mitochondrie en la fusionnant avec la partie C-terminale du peptide signal (22 acides aminés) de la sousunité IV de la cytochrome oxydase mitochondriale. On peut donc, grâce à l'utilisation d'un système de synthèse in vitro, observer la translocation de la protéine hybride ainsi formée (pCoxIV-DHFR) dans les mitochondries. Dans ce genre d'études, la DHFR constitue une sonde très utile, d'autant plus que sa structure tridimensionnelle est connue et peut facilement être évaluée à l'aide de sondes fluorescentes ou en étudiant, par exemple, sa sensibilité à une digestion ménagée par la trypsine. Des études antérieures du groupe de Schatz [1] avaient montré que la présence de la pré-séquence ne change pas la conformation générale de la partie DHFR. On ne peut donc expliquer le phénomène de translocation de la protéine hybride par l'adoption d'une conformation par- ticulière induite par la présence du peptide signal hétérologue.

L'équipe de Schatz avait aussi montré que la translocation de la pCoxIV-DHFR dans la mitochondrie impliquait au moins deux étapes. Dans un premier temps, la protéine se lie à la surface mitochondriale et la partie DHFR est déployée partiellement. Cette étape est lente, stimulée par le potentiel membranaire et inhibée par le méthotrexate (un inhibiteur compétitif de la DHFR) et ralentie à basse température. Elle ne requiert pas d'ATP. Dans un deuxième temps, la protéine partiellement déployée pénètre dans la matrice mitochondriale, perd son peptide signal et la partie DHFR se replie dans sa structure tertiaire native. Cette étape est rapide, dépend de l'ATP, ne nécessite pas de potentiel membranaire. Elle a lieu même à basse température et est insensible au méthotrexate [2]. D'autre part, l'équipe de Schatz avait également observé que les mêmes changements conformationnels de la pCoxIV-DHFR caractéristiques de la première étape de translocation pouvaient être obtenus en utilisant de simples vésicules lipidiques riches en phospholipides acides et exemptes de protéines [1].

Ces résultats ont incité ces chercheurs à vérifier l'importance des phospholipides acides dans l'importation des protéines mitochondriales. Pour ce faire, ils ont utilisé deux approches. La première consiste à vérifier l'effet de l'adriamycine sur la translocation de la pCoxIV-DHFR dans les mitochondries [3]. Cette drogue, qui se lie à la cardiolipine ainsi qu'à d'autres phospholipides acides, s'est avérée capable de bloquer spécifiquement la première étape de la translocation de la pCoxIV-DHFR native dans les mitochondries. Cette inhibition est moindre pour la pCoxIV-DHFR 
comportant trois mutations ponctuelles dans la partie DHFR qui déstabilisent sa structure tertiaire. Cette inhibition est complètement éliminée si la protéine est dénaturée avant de procéder à la translocation. D'autre part, les auteurs ont également vérifié que le déploiement partiel du précurseur par des liposomes riches en cardiolipine est inhibé par l'adriamycine. Il semble donc que l'adriamycine puisse bloquer la translocation du précurseur en empêchant le déploiement du précurseur et que les phospholipides acides participent directement ou indirectement à la translocation.

Dans une deuxième approche [4], les auteurs ont vérifié si l'étape initiale de la translocation de la pCoxIVDHFR pouvait avoir lieu sur des vésicules préparées à partir de membranes externes de mitochondries ou sur des liposomes exempts de protéines et dont la composition en lipides acides est semblable à celle des membranes externes de mitochondrie. Les résultats montrent que la pCoxIV-DHFR se lie à ces deux sortes de vésicules et que cette liaison implique sa dénaturation partielle. Ces résultats permettent aux auteurs de proposer un rôle pour les phospholipides acides de la membrane externe de la mitochondrie lors de l'importation des précurseurs des protéines mitochondriales. Une première réaction consisterait en la for- mation de liens électrostatiques entre le peptide signal chargé positivement et les groupes acides des phospholipides. La seconde réaction impliquerait un changement de conformation de la DHFR qui serait induit par les phospholipides acides (étapes 1 et 2 de la figure 1).

Le mécanisme proposé par le groupe de Schatz ne fait aucunement appel à des constituants protéiques pour assurer la translocation des précurseurs à l'intérieur de la mitochondrie. Cette théorie est cependant en contradiction avec les travaux de deux autres groupes au moins. Ainsi, le laboratoire de Shore a montré que si la séquence signal du précurseur de l'ornithine transcarbamylase pou-

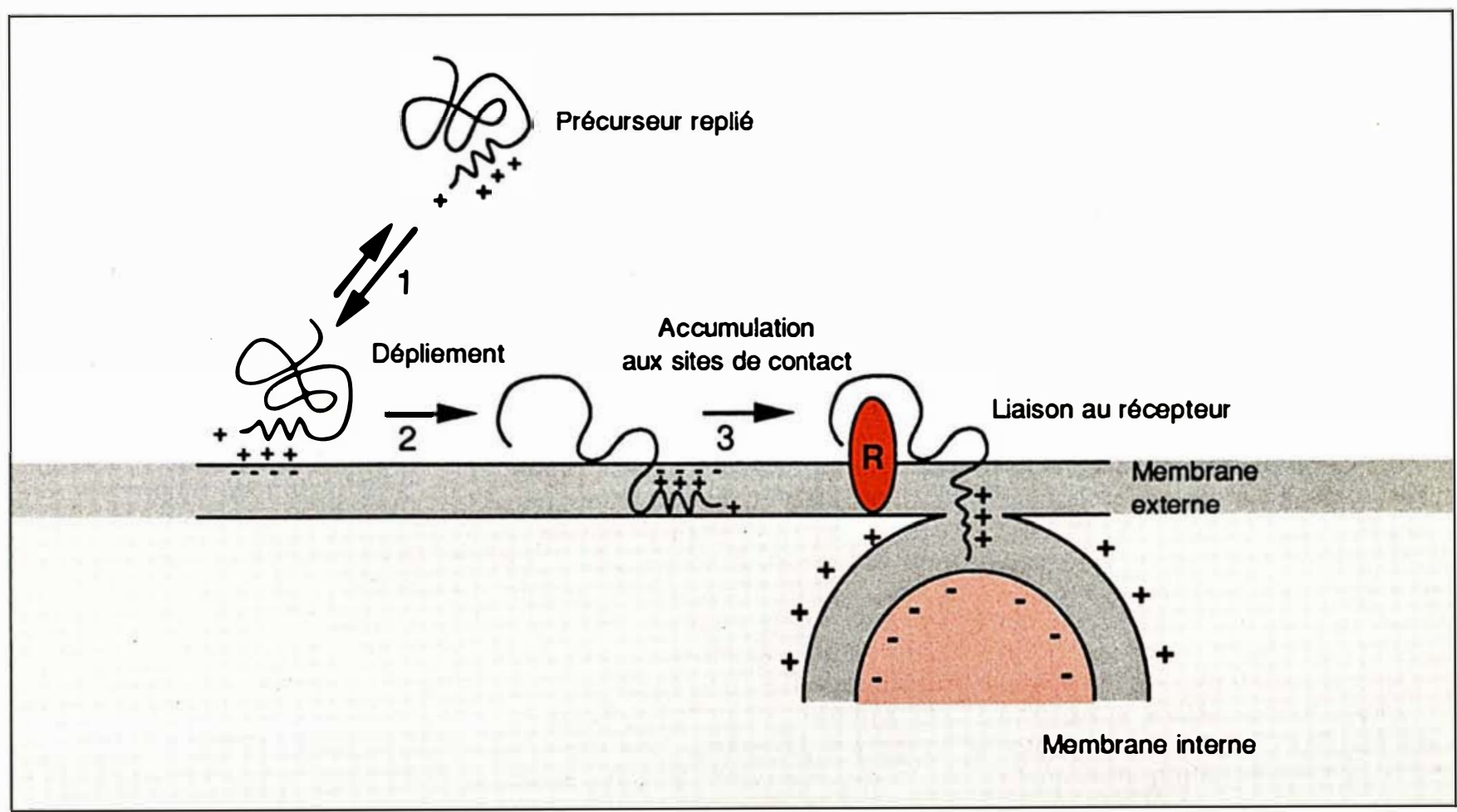

Figure 1. Modèle de translocation des protéines mitochondriales. Les protéines mitochondriales sont synthétisées sous la forme de précurseurs sur des ribosomes libres présents dans le compartiment cytoplasmique de la cellule. Ces précurseurs, qui portent une séquence signal N-terminale additionnelle, sont relâchés dans le cytosol. Les charges positives présentes sur le peptide signal du précurseur favorisent la liaison de ce dernier avec les charges négatives des phospholipides présents sur la membrane externe de la mitochondrie (étape 1). L'interaction du précurseur avec ces charges négatives de la membrane externe provoque alors un changement dans la conformation du précurseur (étape 2). Cette nouvelle conformation favorise la liaison du précurseur avec la machinerie assurant la translocation (étape 3) à l'endroit où les membranes externe et interne se rejoignent. Les composants de cette machinerie sont encore mal connus. II semble cependant que le mécanisme implique l'intervention d'un récepteur (R) chargé de lier le précurseur dans sa nouvelle conformation et d'initier ainsi la translocation elle-même. 
vait faciliter l'interaction initiale de cette protéine avec des vésicules lipidiques, on ne pouvait observer de translocation dans ce système, même en présence d'un potentiel transmembranaire comparable à celui trouvé dans les mitochondries in vivo [5]. Le groupe de Neupert a également trouvé que la digestion des sites récepteurs à la surface mitochondriale par la trypsine réduisait considérablement l'efficacité du mécanisme d'importation de plusieurs protéines mitochondriales [6]. De plus, le faible niveau de translocation résiduelle observé manque de spécificité puisqu'il permet d'importer avec la même très faible efficacité les protéines mitochondriales et le précurseur d'une protéine normalement retrouvée dans les chloroplastes. Selon Shore, l'association des précurseurs avec les lipides membranaires ne servirait qu'à faciliter la liaison ultérieure de ces protéines avec le récepteur spécifique dont l'intervention est nécessaire pour conférer l'efficacité et la spécificité du phénomène d'importation (étape 3 de la figure 1).

Il est également possible qu'un ou plusieurs facteurs cytosoliques puissent être requis pour maintenir les précurseurs des protéines mitochondriales dans une conformation propre à faciliter leur translocation. Jusqu'à présent, ces facteurs n'ont été ni clairement identifiés ni purifiés. Toutefois, des progrès récents réalisés par deux équipes ont permis d'isoler et de caractériser partiellement ces facteurs cytosoliques à partir de lysat de réticulocytes de lapin et de levure. Ono et Tuboi [7] ont étudié le mécanisme de transport des protéines dans la mitochondrie à l'aide d'un peptide synthétique de 34 acides aminés correspondant à la préséquence de l'ornithine aminotransférase. Ce peptide est importé dans la matrice de la mitochondrie de foie de rat et son transport semble s'effectuer de façon identique à celui des protéines mitochondriales normales. L'addition d'un extrait de lysat de réticulocytes augmente significativement le transport du peptide dans la mitochondrie. Cette observation, qui fait écho aux travaux antérieurs du l'existence, dans ce lysat, d'un facteur activant l'importation du peptide. Ce test a donc pu servir de dosage pour suivre la purification du facteur jusqu'à l'obtention d'une fraction dont l'enrichissement a été estimé à 20000 fois par rapport au lysat initial. Même si cette fraction finale n'est pas encore absolument homogène, il semble que le facteur soit de nature protéique, puisqu'il est inactivé par un traitement à la trypsine. En outre, il ne contient pas d'ARN, puisqu'un traitement à la ribonucléase n'a aucun effet. Une caractérisation partielle du facteur permet de proposer qu'il est composé de plusieurs sous-unités et qu'il aurait un poids moléculaire d'environ 200000 . Ono et Tuboi avaient précédemment obtenu une préparation partiellement purifiée contenant un récepteur qu'ils pensent être responsable de l'importation de l'ornithine aminotransférase dans la mitochondrie intacte [9]. Ils ont récemment reconstitué un test de liaison in vitro en incorporant ce candidat récepteur dans des liposomes. A des concentrations de peptide synthétique inférieures à $1,5 \times 10^{-8} \mathrm{M}$, ces liposomes lient une quantité appréciable du peptide synthétique, pourvu qu'on ajoute le facteur cytosolique au milieu d'incubation. A des concentrations du peptide supérieures à $1,5 \times 10^{-8} \mathrm{M}$, cependant, cette interaction ne requiert pas la présence du facteur car le peptide se lie de façon non spécifique aux lipides. Comme la concentration des protéines précurseurs dans le milieu cellulaire n'excède probablement pas $1,5 \mathrm{x}$ $10^{-8} \mathrm{M}$, le facteur cytosolique semblerait donc requis pour assurer l'interaction d'un précurseur de protéines mitochondriales avec son récepteur tant in vitro qu'in vivo. Dans ce dernier cas cependant, l'existence de ce mécanisme reste à prouver de façon directe. De plus les auteurs ne démontrent pas que cette liaison du peptide synthétique au récepteur présumé est l'étape préliminaire nécessaire à l'accomplissement du phénomène d'importation.

De leur côté, Murakami et ses collaborateurs [10] ont utilisé un système traductionnel de germe de blé et une fraction cytosolique de levure appe- lée PRS (post ribosomal supernatant) pour suivre la translocation de la delta-pyrroline-5-carboxylate déshydrogénase (Put2). Comme c'était le cas pour le peptide synthétique calqué sur la pré-séquence de l'ornithine aminotransférase, le transport de Put2 dans la mitochondrie est stimulé par un facteur contenu dans le PRS de levure. L'utilisation du système de la levure a cependant l'avantage supplémentaire de permettre l'introduction des techniques de la génétique pour caractériser de façon précise le gène correspondant aux facteurs identifiés par les études biochimiques. Deshaies et al. [11] ont ainsi démontré que l'inactivation de certains gènes de la famille des protéines induites lors d'un choc thermique (heat shock proteins) provoque l'accumulation in vivo des précurseurs des protéines mitochondriales codées par des gènes nucléaires. Fait remarquable, les mêmes mutations affectaient également la translocation des protéines de sécrétion dans le cytoplasme de levure. Les gènes impliqués codent pour la classe des protéines du choc thermique dont la masse moléculaire est de 70000 (hsp70). De plus, Chirico et al. [12] ont démontré qu'un mélange des protéines hsp70 issues du cytosol de levure, soit Ssalp/Ssa2p, stimule la translocation des protéines à travers les microsomes. D'après les travaux de Murakami et al. [10], Ssalp/Ssa2p stimule également de façon significative le transport de Put2, mais seulement en présence de PRS de levure. Le facteur du PRS semble bien être une protéine, puisque son action est sensible à la chaleur et à la trypsine. Mais alors que la protéine Ssalp/S sa2p est insensible au N-éthylmaléimide (NEM), l'activité stimulatrice du PRS est inhibée par le NEM et contiendrait donc un groupe sulfhydryle nécessaire à son activité.

Ces résultats permettent donc de proposer deux hypothèses: (1) les protéines du choc thermique Ssalp/Ssa2p semblent importantes pour assurer la translocation des protéines destinées à la sécrétion comme de celles destinées aux mitochondries; (2) le facteur cytosolique identifié dans le PRS de levure est distinct de 
Ssalp/Ssa2p mais semble tout aussi nécessaire à la translocation, au moins en ce qui concerne les protéines mitochondriales.

Le groupe de Murakami [6] propose que le facteur sensible au NEM pourrait ressembler au SRP (signal recognition particle) (particule de reconnaissance du peptide signal qui est essentielle à la translocation dans le réticulum endoplamisque rugueux) ( $\mathrm{m} / \mathrm{s} n^{\circ} 6$, vol. $\left.2, p .341\right)$. Tout comme le SRP, il serait chargé de reconnaître et de lier la séquence signal des précurseurs de protéines mitochondriales pour assurer par la suite leur transport du cytoplasme vers la mitochondrie. L'interaction du facteur sensible au NEM avec le récepteur sur la membrane mitochondriale externe causerait la dissociation entre le facteur et la séquence signal de façon que cette séquence interagisse avec un autre récepteur situé cette fois dans la zone de contact entre les membranes externe et interne. Les auteurs n'ont malheureusement pas apporté la preuve directe que le facteur sensible au NEM se lie à la séquence signal des précurseurs mitochondriaux. En l'absence d'une telle preuve, ce mécanisme reste une hypothèse séduisante dont la confirmation permettrait d'expliquer par une théorie unificatrice deux mécanismes de translocation dans des organites différents : le réticulum endoplasmique et la mitochondrie.

L'ensemble de ces travaux semble donc démontrer que l'établissement de la structure tertiaire de protéines destinées à être transférées dans la membrane d'un organite cible pourrait être contrôlé par des facteurs cytosoliques. Cette structure pourrait aussi être modifiée dans une étape préliminaire à la translocation par l'action de certains lipides membranaires chargés négativement

\footnotetext{
Philippe Crine

Nathalie Antoine

Julie Beaudet

Département de biochimie, université de Montréal, case postale 6128, Succ. A, Montréal, H3C 3J7, Québec, Canada

$\mathrm{m} / \mathrm{s} n^{\circ} 9$ vol. 5, novembre 89
}

\section{RÉFÉRENCES}

l. Endo T, Schatz, G. Latent membrane perturbation activity of a mitochondrial precursor protein is exposed by unfolding. EMBO J $1988 ; 7: 1153-8$

2. Eilers M, Schatz G. Protein unfolding and the energetics of proteins translocation across biological membranes, Cell 1988; 52: 481-3.

3. Eilers M, Endo T, Schatz G. Adriamycin, a drug interacting with acidic phospholipids, blocks import of precursor proteins by isolated yeast mitochondria. J Biol Chem 1989; $264: 2945-50$

4. Endo T , Eilers M, Schatz G. Binding of a tightly folded artificial mitochondrial precursor protein to the mitochondrial outer membrane involves a lipid-mediated conformational change. J Biol Chem 1989; 264 : 2951-6.

5. Skerjanc IS, Shore GC, Silvius JR. The interaction of a synthetic mitochondrial signal peptide with the lipid membrane is independant of transbilayer potential. $E M B O$ J $1987 ; 6$ : 3117-23.

6. Pfaller R, Pfanner N, Neupert W. Mitochondrial protein import. Bypass of proteinaceous surface receptors can occur with low specificity and efficiency. J Biol Chem 1989 $264: 34-9$.

7. Ono $\mathrm{H}$, Tuboi S. The cytosolic factor required for import of precursors of mitochondrial proteins into mitochondria. J Biol Chem 1988; $263: 3188-93$.

8. Argan C, Lusty CJ, Shore GC. Membrane and cytosolic components affecting transpor of the precursor for ornithine carbamyltransferase into mitochondria. J Biol Chem 1983 ; $258: 6667-70$

9. Ono $\mathrm{H}$ Tuboi $\mathrm{S}$. Partial purification of the receptor protein essential for import of the ornithine aminotransferase into mitochondria. Biochem Int 1985; 10 : 351-7.

10. Murakami H, Pain D, Blobel G. 70 kDa heat shock-related protein is one of at least two distinct cytosolic factors stimulating protein import into mitochondria. I Cell Biol $1988 ; 107: 2051-7$.

11. Deshaies RJ, Koch BD, Werner-Washburne M, Craig EA, Scheckman R. A subfamily of stress proteins facilitates translocation of secretory and mitochondrial precursor polypeptides. Nature $1988 ; 332: 800-5$.

12. Chirico WJ, Waters MG, Blobel G. 70K heat shock related proteins stimulate protein translocation into microsomes. Nature 1988 $332: 805-10$. 